\title{
LA TÉCNICA DEL ROSAL COMO DIAGNÓSTICO EMOCIONAL EN POBLACIONES ESPECÍFICAS
}

\section{ROSAL TECHNIQUE AS AN EMOTIONAL DIAGNOSIS IN SPECIFIC POPULATIONS}

\author{
Núria Garcia y Nathalie P. Lizeretti \\ Centro de Investigación, Formación y Desarrollo de la Inteligencia Emocional. \\ Fundación Ramón Rosal Cortés, Barcelona, España
}

Cómo referenciar este artículo/How to reference this article:

Garcia, N. y Lizeretti, N.P. (2017). La técnica del rosal como diagnóstico emocional en poblaciones específicas. Revista de Psicoterapia, 28(107), 191-205.

\section{Resumen}

Las técnicas de fantasía guiada suponen una gran fuente de información diagnóstica (Rosal, 2002), que permite un trabajo terapéutico creativo al tiempo que constituyen estrategias efectivas para el desarrollo de la Inteligencia Emocional (Lizeretti, 2009; 2012). El objetivo del presente estudio ha sido sistematizar el análisis de la técnica del rosal a fin de contribuir a su validez empírica en el proceso psicodiagnóstico. Para ello se presentan de forma esquemática dos estudios donde se ha aplicado la técnica desde el modelo de Terapia basada en Inteligencia Emocional (TIE). El primer estudio en una muestra de 92 participantes, pacientes con ansiedad y depresión clínica derivados de un centro de salud mental y un grupo control sin diagnostico. El segundo estudio estuvo formado por 62 menores, un grupo experimental procedente de Centros Residenciales de Acción Educativa (CRAE's) y un grupo control procedente de escuelas de secundaria. Los resultados muestran diferencias claramente significativas entre los diferentes grupos que se han comparado, demostrando la importancia de la investigación empírica en este ámbito a fin de consolidar la eficacia de las técnicas de fantasias guiadas y proyectivas.

Palabras clave: Inteligencia Emocional, Depresión, Ansiedad, Menores, CRAES, Técnicas de fantasia guiada, Terapia basada en Inteligencia Emocional (TIE).

\begin{abstract}
Guided fantasy techniques provide a great source of diagnostic information (Rosal, 2002), which allows creative therapeutic work and are effective strategies for the development of Emotional Intelligence (Lizeretti, 2009, 2012). The aim of the present study was to systematize the analysis of the rosal technique in order to contribute to its empirical validity in the psychodiagnostic process. For this, two studies are presented schematically where the technique has been applied from the model of Therapy based on Emotional Intelligence (TIE). The first study with a sample of 92 participants, patients with anxiety and clinical depression derived from a mental health center and a control group without diagnosis. The second study consisted of 62 minors, an experimental group from Residential Educational Action Centers (CRAE's) and a control group from secondary schools. The results show clearly significant differences between the different groups that have been compared, demonstrating the importance of empirical research in this area in order to consolidate the effectiveness of guided and projective fantasy techniques.
\end{abstract}

Keywords: Emotional Intelligence, Depression, Anxiety, Minors, CRAES, Guided Fantasy Techniques, Therapy based on Emotional Intelligence (TEI).

Fecha de recepción: 11 de mayo de 2017. Fecha de aceptación: 17 de junio de 2017.

Correspondencia sobre este artículo:

E-mail: nuriagarcia@cidie.es

Dirección postal: C/ Pintor Veláquez, 66, 08302 Mataró, Barcelona, España

(C) 2017 Revista de Psicoterapia 
La Fantasía del Rosal es una técnica gestáltica de fantasía guiada que enfatiza en el darse cuenta awarness como agente curativo, propiedad que toda persona tiene y que tal como señala Stevens (1976) se va perdiendo con la edad. Por ejemplo, el bebé se limita a algunas necesidades emocionales y fisiológicas como lo que es grato e ingrato, que expresa en forma de sonrisa o llanto para comunicar estos estados emocionales agradables o desagradables. A media que vamos creciendo aumenta la capacidad de darse cuenta de estos estados emocionales y necesidades biológicas, como sentir una emoción u otra, tener o no tener hambre, etc. Pero esto que resulta tan sencillo puede complicarse a medida que nos relacionamos con el entorno y nos integramos con él, a partir de las relaciones sociales, estilos educativos, personalidad, expectativas y deseos, proyecto de vida, etc. Por lo que empezamos a confundir entre lo que uno es y lo que la sociedad espera de él, entre lo que uno quiere y lo que quieren los demás, entre lo que es real y lo que no.

En la actualidad existe en psicoterapia un creciente interés por técnicas de fantasía guiada, tanto para el diagnóstico como para el tratamiento. Aunque se encuentran pocas investigaciones sobre técnicas de imaginación y fantasía guiada, las existentes demuestran que constituyen una valiosa fuente de información diagnóstica que además generan cambios en el "aquí y ahora" (para una revisión véase Rosal, 2002, p.334). La fantasía guiada permite aflorar material no consciente o explorar aspectos desconocidos, permitiendo el cierre simbólico de situaciones imposibles de cerrar en la realidad. Por otro lado, en la adaptación realizada por Lizeretti $(2009$; 2012) sugiere que permite un trabajo creativo con la dimensión emocional, pudiendo identificar las necesidades básicas asociadas a estados emocionales, constituyen estrategias efectivas para el desarrollo de la Inteligencia Emocional, permitiendo captar el sufrimiento de manera más pura y auténtica que los test psicométricos, y recogiendo información que es muy difícil de obtener de otra manera, o se tardaría mucho.

La Inteligencia Emocional (IE) se ha convertido en los últimos años en un concepto que ha despertado gran interés tanto entre los científicos como entre la población general. Intuitivamente se podría definir como "la competencia para utilizar la sabiduría natural que aportan las emociones" (Lizeretti, 2012 p.134). La IE se compone de cuatro niveles de habilidades emocionales descritas como "la habilidad para percibir con precisión, evaluar y expresar emociones; la habilidad de acceder y/o generar sentimientos cuando facilitan el pensamiento; la habilidad de entender la emoción y el conocimiento emocional; y la habilidad de regular las emociones para fomentar el crecimiento intelectual y emocional" (Mayer y Salovey, 1997 p.10). Este modelo es el más aceptado por la población científica por sistematizar el procesamiento cognitivo de la experiencia emocional, lo que ofrece un marco excepcional para el trabajo terapéutico con las emociones en psicoterapia (Lizeretti y Gimeno-Bayón, 2014).

La IE se asocia con la felicidad, afecto positivo, satisfacción vital y autoestima (Gohm y Clore, 2002). Las personas con altas puntuaciones en IE son más 
optimistas, tienen más autoestima, presentan una mayor capacidad empática (Bracket y Mayer, 2003; Extremera y Fernández-Berrocal, 2004; Mayer, Caruso y Salovey, 1999; Salovey, Stroud, Woolery y Epel, 2002; Schutte, Malouff, Hall, Haggerty, Cooper, Golden, C y Dornheim, 1998) y muestran más conductas prosociales (Mayer et al., 1999), lo que contribuye a un mayor bienestar psicológico. En cambio, cuando es baja se relaciona con conductas desviadas y pobres relaciones sociales (Bracket, Mayer y Warner, 2004). Altos niveles de atención a las emociones, una baja claridad y reparación emocional está vinculado a síntomas como la ansiedad (Salguero y Iruarrizaga, 2006) o la depresión (FernándezBerrocal, Extremera y Ramos, 2004; Augusto-Landa, López-Zafra, Martínez de Antoñana y Pulido Martos, 2006). La IE también tiene un impacto significativo en el ajuste psicológico de los adolescentes asociándose negativamente a la salud mental, a los comportamientos agresivos y perturbadores (Bracket et al., 2004; Davis y Humphrey, 2012; Kokkinos y Kipritsi, 2012; Lomas, Stough, Hansen y Downey, 2012; Mayer, Roberts y Barsade, 2008; Moriarty, Stough, Tidmarsh, Eger y Dennison, 2001), y positivamente a la empatía, las relaciones personales y la satisfacción con el entorno social (Ciarrochi, Chan y Caputi, 2000; Mayer et al., 1999). Además la IE está relacionada con la presencia de los trastornos mentales indicando que los pacientes con psicopatología presentan menos IE que la población general (Jacobs et al., 2008; Lizeretti, Extremera y Rodriguez, 2012; Perna et al., 2010). Por ejemplo, existe relación entre el nivel de IE y la presencia de TAG (Lizeretti y Extremera, 2011) y depresión (Downey, Mountstephen, Lloyd, Hansen y Stough, 2008; Hertel, Schütz y Lammers, 2009).

En este sentido, podemos considerar la IE como un factor de protección de la salud mental y de los trastornos psicopatológicos. Por este motivo, se quiere destacar que desde este modelo de Inteligencia Emocional se puede contribuir a explicar los procesos emocionales que se dan en las diferentes psicopatologías, a fin de comprender mejor su etiología y poder intervenir de una forma más eficaz. El abordaje psicoterapéutico de estas habilidades emocionales puede ser el foco del tratamiento en el abordaje de los trastornos mentales. A pesar de que las investigaciones que estudian el concepto de IE en el ámbito de la psicopatología clínica son aun limitadas, los resultados que se han obtenido hasta el momento señalan que se encuentran ante un nuevo paradigma desde donde formular modelos explicativos para los diferentes trastornos mentales. También pueden contribuir al diseño y aplicación de programas de intervención psicológica eficaces basados en el desarrollo de estas habilidades. En la Terapia basada en Inteligencia Emocional. Manual de tratamiento (Lizeretti, 2012) se ofrecen herramientas para el diagnóstico y la intervención de los diferentes problemas emocionales, una de ellas la Técnica del Rosal (Stevens, 1976; adaptación Lizeretti, 2009).

En este sentido la Técnica del Rosal (Stevens, 1976) no solo facilita la imaginación y el cambio creativo, sino que también sirve como diagnóstico fenomenológico (Rosal, 2002), constituye un test proyectivo con el fin de aflorar 
material no consciente e identifica aspectos relevantes de la personalidad. En su adaptación (Lizeretti, 2009) identifica la emoción vital básica a través de la fantasía y del dibujo, así como los aspectos inconscientes de la personalidad que están relacionados.

Esta técnica facilita una intervención creativa que permite poner de manifiesto las emociones generadoras de los problemas y conflictos emocionales tanto a nivel personal como relacional. Pero además tal y como indica Rosal (1983), la actitud creadora da lugar a un estilo peculiar en el modo de percibir, pensar, emocionarse, motivarse, decidir, de carácter muy personal, idiosincrásico, fiel a uno mismo. La experiencia creadora requiere, más que unos rasgos característicos de la personalidad, una vivencia más o menos profunda de encuentro con uno mismo y con el otro.

\section{La Técnica del Rosal desde el modelo Terapia basada en Inteligencia Emocional (TIE).}

En esta nueva versión de la técnica se han realizado algunas adaptaciones como la reducción de la fantasía, el cambio de objetivo y algunos aspectos fundamentales de la interpretación. Tal y como se ha mencionado anteriormente, el objetivo principal en esta adaptación es identificar la emoción vital básica a través de la fantasía y del dibujo, así como aspectos inconscientes de la personalidad (Lizeretti, 2012).

Desde el modelo de la TIE las emociones sirven como guía y nos orientan hacia el descubrimiento de la necesidad asociada a dicha emoción. Cuando la emoción es auténtica actúa de forma emergente satisfaciendo las necesidades vitales y contribuyendo al bienestar-aquel que nos ayuda a recuperar el equilibrio alcanzando una experiencia auténtica de comodidad o bienestar-. Pero también contribuye al bienser a través de la satisfacción de aquellas necesidades que nos dan una identidad y nos acercan a todo aquello que podemos llegar a ser, dando sentido a nuestra vida, logrando objetivos vitales e integrando nuestras vivencias pasadas, presentes y futuras. Para lograr un auténtico estado de bienestar es necesario que se oriente hacia el bienser. Sin ello, el bienestar es superficial y fugaz y no sirve como una experiencia auténtica de felicidad, equilibrio o paz interior (Lizeretti, 2012 p. 28). Cuando se actúa desde esta superficialidad y el ciclo de la experiencia emocional queda incompleto, ya sea porque se ha bloqueado, dispersado o distorsionado la energía generada por la emoción, imposibilita satisfacer la necesidad auténtica, provocando malestar e insatisfacción. En este caso la emoción queda prohibida, pero la energía emocional necesita una vía de escape, y por ello, cuando prohibimos una emoción, la energía se deriva hacia otra emoción (parásita) motivada a la satisfacción de otras necesidades por lo que las necesidades asociadas a la emoción prohibida quedan insatisfechas. Por ejemplo, imaginemos una persona que desde pequeña se le ha prohibido la emoción de la tristeza a través de mensajes como "llorar es de niñas, tienes que ser fuerte, no seas débil", esta persona para satisfacer 
las expectativas del entorno aprenderá a no mostrar o expresar esta emoción aunque la sienta, sin embargo, desviará toda esta energía hacia otra emoción, que puede ser, por ejemplo, la rabia parásita. De tal modo que ante una situación de tristeza, como una pérdida, la persona se verá motivada a expresar conductas agresivas, disruptivas, de enfado, etc. para intentar tapar los sentimientos de vacío. Pero quedará insatisfecho al no recibir el consuelo que necesita (necesidad vital básica ante la pérdida) lo que le llevará a sentimientos de culpabilidad, resentimiento o frustración (a corto y largo plazo). Cuando la persona aprende a expresar la emoción auténtica y satisfacer la necesidad que la desencadena puede cerrar el ciclo de la experiencia emocional reparando sus estados emocionales desagradables.

Teniendo en cuenta la importancia de identificar de forma adecuada las emociones que subyacen a nuestras experiencias emocionales y las necesidades vitales vinculadas a la emoción auténtica, desde este modelo se lleva a cabo un diagnóstico emocional. La comprensión e información que aporta el diagnóstico emocional sirve como guía para el diagnóstico psicopatológico y puede resultar útil para formular la teoría del problema en el contexto psicoterapéutico. En este enfoque se atiende tanto al proceso como al contenido de la emoción, y el diagnóstico del proceso emocional se prioriza sobre el diagnóstico psicopatológico. La formulación del problema en términos de habilidades emocionales es útil para identificar emociones prohibidas y parásitas en torno a las que se construye el problema y ayuda a ajustar la tarea terapéutica a las necesidades auténticas del paciente, ayudando al establecimiento de objetivos eficaces. Este diagnóstico es una alternativa y complementa a los diagnósticos tradicionales, ya que permite hacer un análisis emocional exhaustivo, tiene en cuenta el perfil de personalidad en la que se gesta y desarrolla el síntoma. Además hace referencia al proyecto vital de la persona y tiene una visión sistémica donde identificar patrones relacionales disfuncionales, con el objetivo principal de comprender de forma holista el sufrimiento de la persona. El diagnóstico emocional abre la posibilidad de intervenciones más creativas orientadas no solo a la solución de problemas sino también al crecimiento personal (Lizeretti, 2012 p.103). Por todo ello se ha considerado importante validar empíricamente, no sólo la eficacia de la Terapia basada en Inteligencia Emocional (Lizeretti, 2009) sino también iniciar un proceso de validación de las diferentes técnicas y estrategias de intervención empleadas. En el presente trabajo se presentan algunos de los primeros resultados obtenidos en el proceso de validación de la Técnica del Rosal.

\section{Método}

Participantes. Para llevar a cabo esta investigación se realizaron 2 estudios. El primer estudio se realizó con una muestra de 95 participantes compuesta por 30 pacientes diagnosticados de trastorno de ansiedad y 21 con diagnóstico de depresión derivados de un Centro de Salud Mental de la provincia de Barcelona, y un Grupo Control (GC), muestra de la población general $(n=44)$. El 79\% de los 
participantes eran mujeres y la media de edad fue de 41 años $(\mathrm{DT}=14,5)$.

En el segundo estudio participaron 62 menores de ambos sexos con una media de edad de 13,60 años (DT=2,75). Un grupo experimental de 36 menores procedentes de Centros Residenciales de Acción Educativa (CRAE's) tutelados por la Direcció General d'Atenció a la Infància i Adolescència (DGAIA) de la Generalitat de Catalunya y un Grupo Control (GC) formado por 26 menores procedentes de diferentes centros escolares de Catalunya.

Los participantes fueron informados de los propósitos de la investigación y expresaron su participación voluntaria en el estudio a través del documento de consentimiento informado.

Material. El material empleado fue el procedimiento de la Técnica del Rosal (Lizeretti, 2009) que empieza por un ejercicio de fantasía guiada con una previa relajación. Tras la breve fantasía se procede a elaborar el dibujo y la descripción del rosal con las principales características. Seguidamente se realiza la interpretación en colectivo donde se identifican la emoción principal que les genera el dibujo, emoción parásita y la emoción que sentirían al ser este rosal, emoción prohibida, así como otros aspectos relevantes del dibujo (las necesidades básicas en torno a la que se construye la personalidad y el problema). De tal manera que, como en otras técnicas proyectivas, la interpretación se basa en la lógica emocional y en el sentido común.

Procedimiento. La técnica fue aplicada en la primera sesión de terapia grupal porque además aportar una valiosa información en el proceso de diagnóstico emocional, permite dar a conocer la dinámica que guiará la intervención terapéutica, la participación de todos los miembros del grupo en el proceso terapéutico, compartir aportaciones, facilitar el propio cambio y el del resto del grupo.

Para llevar a cabo la validación del proceso de interpretación del dibujo del rosal se llevaron a cabo los siguientes pasos: Primero se establecieron en categorías los criterios utilizados para la interpretación de las pruebas proyectivas del siguiente modo: tamaño, ubicación en la hoja, trazo, color, etc. Posteriormente se hizo lo mismo con los elementos del rosal (raíces, tronco, espinas, hojas, flores y ramas) y los elementos del entorno (árbol, casa, maceta, montañas, sol, nubes, etc.). También se analizó y recogió la emoción auténtica y parásita de cada participante. Finalmente se sistematizaron las categorías en diferentes variables con el objetivo de encontrar resultados lo más fiables posibles para su empleo diagnóstico.

En el proceso de sistematización de los datos y su análisis participaron diferentes profesionales expertos en Terapia Basada en Inteligencia Emocional y formados en el diagnóstico emocional. Como procedimiento para la estimación de la fiabilidad de las categorías se utilizó el método de evaluación interjueces. Para el análisis estadístico se llevaron a cabo análisis de frecuencia y tablas de contingencia. 


\section{Resultado}

Este artículo no pretende hacer un análisis exhaustivo de todos los resultados obtenidos en estos estudios sino ofrecer algunos de los resultados más significativos que ayudan a demostrar por una parte, la riqueza que puede aportar esta técnica y por otra, a demostrar cómo la sistematización en su interpretación puede contribuir a su validez empírica como herramienta en el proceso psicodiagnóstico. Además de destacar la necesidad de investigaciones científicas en este campo que demuestren y consoliden la eficacia de las técnicas de fantasías guiadas y proyectivas.

Estudio 1. A continuación se muestran algunos de los resultados más significativos del primer estudio.

- Diferencias entre población general y población clínica

Se encuentran diferencias significativas entre la población clínica y el grupo control $\left(\mathrm{X}^{2}=, 002\right)$ en cuanto a la emoción parásita. La mayoría de las personas del GC expresaron alegría $(45,5 \%)$ como emoción parásita, en cambio la población clínica expresó en mayor medida el miedo $(43,1 \%)$ o la tristeza $(31,4 \%)$. En relación a la emoción auténtica, la mayor parte de la población general $(75 \%)$ presentó el miedo como emoción auténtica. Entre la población clínica el $52,9 \%$ presentó miedo y el $45,1 \%$ rabia.

- Diferencias entre diagnósticos

Cuando se comparan grupos diagnósticos se observan diferencias significativas entre las personas con depresión y ansiedad respecto a la emoción parásita $\left(\mathrm{X}^{2}=, 001\right)$. Las personas con depresión expresan tristeza como emoción parásita $(57,11 \%)$ y las personas con ansiedad el miedo (46,7\%). Pero curiosamente al analizar la emoción auténtica se observa que las personas con depresión en realidad lo que tienen es miedo en el $66,7 \%$ de los casos y rabia el $50 \%$ de las personas con ansiedad.

Más concretamente, los resultados indican que las personas con Trastorno de Ansiedad Generalizada (TAG) presentan la alegría como emoción parásita $(45,5 \%)$ y las personas con agorafobia el miedo $(63,2 \%)$, dándose diferencia significativas entre ellas $\left(\mathrm{X}^{2}=, 006\right)$. En cuanto a la emoción prohibida, las personas con TAG presentan miedo $(66,7 \%)$ y las personas con agorafobia rabia $(57,9 \%)$, aunque esta diferencia no es estadísticamente significativa $\left(X^{2}=, 110\right)$.

$\mathrm{Al}$ analizar los elementos constitutivos del rosal se encontraron diferencias entre ambos diagnósticos. Por ejemplo, las personas con depresión $(57,9 \%)$ al dibujar el rosal no conectan el tronco con otros elementos como las raíces o las ramas, en cambio la mayoría de los pacientes con ansiedad $(79,3 \%)$ hacen dibujos en los que todos los elementos están bien conectados, siendo estas diferencias significativas $\left(\mathrm{X}^{2}=, 009\right)$. Asimismo, se observan diferencias respecto al nivel de conexión entre los pacientes con Agorafobia y las personas con TAG $\left(X^{2}=, 008\right)$. En la agorafobia $(89,5 \%)$ parece haber más conexión que en el TAG $(60 \%)$

La posición del rosal en el lienzo también se tomó como indicador. El 52, 4 \% de los pacientes con depresión dibujan el rosal en la parte superior, el $45 \%$ de los 
pacientes con TAG lo dibujan en la parte de abajo y el $63,2 \%$ de las personas con agorafobia en el centro, dándose una diferencia significativa $\left(X^{2}=, 025\right)$.

En los rosales de los pacientes con ansiedad $(85,7 \%)$ se observa el uso de colores que corresponden a la realidad (por ejemplo las rosas son de color rosa o rojo) pero no así en los pacientes con depresión que con frecuencia $(58,3 \%)$ utilizan colores poco fieles a la realidad (azul, negro, marrón o lila), siendo este dato también significativo $\left(\mathrm{X}^{2}=, 042\right)$.

Las espinas están relacionadas con los mecanismos de defensa de las personas. La mayoría de los pacientes con depresión $(42,97 \%)$ tienden a no dibujar espinas, mientras que los pacientes con ansiedad $(66,7 \%)$ si lo hacen, indicando otra diferencia entre estos dos grupos diagnósticos $\left(\mathrm{X}^{2}=, 005\right)$.

- Diferencias respecto a la emoción parásita

Un dato que ha resultado de especial interés y muy significativo $\left(X^{2}=, 000\right)$ indica que todas las personas que expresan rabia (100\%) tienen miedo como emoción auténtica. Por el contrario, todos los que expresan miedo $(100 \%)$ tienen la rabia como emoción prohibida. Las personas que expresan tristeza $(88,8 \%)$, tienen miedo auténtico del mismo modo que las que expresan alegría $(71,4 \%)$.

$\mathrm{Al}$ analizar el tipo de entorno se encontraron diferencias significativas $\left(\mathrm{X}^{2}=\right.$ ,010), en cuanto a la emoción parásita. Las personas que expresan alegría $(60 \%)$ han dibujado un entorno afable (flores, ríos, montañas), las que expresan miedo $(66,6 \%)$ dibujan un entorno hostil (borrasca, rayos) así como las que expresan rabia (50\%). La otra mitad dibujan un entorno vacío (50\%) junto los pacientes que han expresado tristeza $(47,1 \%)$. Seguramente porque la tristeza es más introspectiva y focaliza menos en el entorno, pero cuando se fija lo ve hostil $(41,7 \%)$.

Las mayoría de personas que expresan alegría $(71 \%)$ y miedo $(88 \%)$ han dibujado el rosal nutrido, es decir con color, flores abiertas, frondosos, etc. En cambio las personas que expresan tristeza $(72 \%)$ y rabia $(50 \%)$ han dibujado un rosal desnutrido, por ejemplo con la tierra seca, el tronco negro, las hojas y flores mustias, etc.. apreciándose diferencias significativas $\left(X^{2}=, 010\right)$. Respecto al número de flores, las personas que expresan tristeza (70\%) sólo han dibujado una flor y las personas que transmiten rabia $(50 \%)$ han dibujado más de 5 , hallándose una diferencia significativa entre ambos grupos $\left(\mathrm{X}^{2}=, 045\right)$.

- Diferencias respecto a la Emoción Auténtica:

También se encuentran diferencias significativas respecto a la presencia de espinas $\left(X^{2}=, 049\right)$, mostrando que todas las personas con tristeza auténtica $(100 \%)$ no dibujan espinas en el rosal y presentan desprotección. Las personas que sienten rabia (77\%) dibujan espinas, de las cuales un $68,4 \%$ lo hacen como defensa. Asimismo, las personas con miedo auténtico (55\%) dibujan espinas, y el 30\% lo hacen como defensa.

- Diferencias entre sexos

$\mathrm{Al}$ analizar la emoción parásita en relación a la variable sexo se encontraron diferencias significativas entre ambas variables $\left(X^{2}=, 029\right)$, indicando que los 
hombres expresan rabia (37,5\%) o miedo (50\%), como emoción parásita y las mujeres tristeza $(37,2 \%)$ y miedo $(32,6 \%)$.

Estudio 2. Los resultados más significativos del estudio 2 son:

- Diferencias entre el grupo experimental y el grupo control

Cuando comparamos grupos diagnósticos se observan diferencias significativas $\left(X^{2}=, 003\right)$ entre el GC que presenta alegría como emoción parásita $(14,5 \%)$ y los menores tutelados que presentan rabia $(27,4 \%)$.

De los elementos constitutivos del rosal, las hojas son un indicador relacionado con los recursos personales que puede tener la persona. Resulta interesante observar como los menores tutelados no dibujan hojas en sus rosales $(37,1 \%)$, pero si lo hacen los menores del GC $(37,1 \%)$ observándose diferencias significativas $\left(\mathrm{X}^{2}=, 019\right)$ entre ambos grupos. Además, el GC presenta un rosal nutrido $(35,5 \%)$, en cambio el grupo experimental presenta el rosal desnutrido (27,7\%). En la misma línea, los menores del GC dibujan un entorno afable $(13,1 \%)$ o neutro $(18 \%)$, por el contrario los menores tutelados presentan un entorno hostil (23\%) observándose diferencias significativas $\left(\mathrm{X}^{2}=, 006\right)$ entre ambos grupos diagnósticos.

- Diferencias respecto al abuso o maltrato

Otro dato interesante del que se dispone información y se han hallado diferencias altamente significativas $\left(\mathrm{X}^{2}=, 000\right)$ ha sido el maltrato (físico y/o psicológico y el abuso sexual) en relación al grupo diagnóstico. Ninguna persona del GC presenta abuso ni maltrato y sólo un 1,7\% presenta sospecha. En cambio, entre menores tutelados hay un alto índice de maltrato (30\%) y de abuso sexual $(11,7 \%)$ así como de sospecha $(13,3 \%)$.

En relación con la emoción parásita se observan diferencias significativas $\left(X^{2}=, 026\right)$ en cuanto al tipo de maltrato. Los niños que han sufrido abuso sexual expresan tristeza $(8,3 \%)$ y rabia $(3,3 \%)$ como emoción parásita, los niños que han sufrido maltrato expresan rabia $(13,3 \%)$ y los niños con sospecha miedo $(6,7 \%)$.

La mayoría de los menores que han sufrido abuso o maltrato $(23,2 \%)$ no han dibujado el rosal con colores fieles a la realidad, en cambio los menores que no han sufrido maltrato $(31,6 \%)$ lo dibujan con colores fieles a la realidad, observándose diferencias significativas $\left(\mathrm{X}^{2}=, 007\right)$. Así mismo, los menores que han sufrido abuso sexual presentan un rosal desnutrido (10\%) igual que las personas con sospecha $(8 \%)$, en cambio los que no han sufrido ningún tipo maltrato $(36,7 \%)$ presentan un rosal nutrido, dándose diferencias significativas $\left(X^{2}=, 004\right)$ entre estas variables.

- Diferencias entre sexos

Se observan diferencias significativas $\left(\mathrm{X}^{2}=, 025\right)$ en relación al sexo y algunos de los elementos del rosal. Por ejemplo, los chicos han dibujado más espinas en su rosal $(25,9 \%)$ y ninguna hoja $(27,4 \%)$. En cambio las chicas no dibujan espinas $(38,7 \%)$ pero si hojas $(37,1 \%)$.

- Diferencias respecto la emoción parásita

Las personas con alegría parásita (10\%) dibujan más de 5 flores en su rosal. Las personas que expresan rabia $(21,7 \%)$, tristeza $(11,7 \%)$ y miedo $(16,7 \%)$ dibujan 
solo una flor, observándose diferencias significativas $\left(\mathrm{X}^{2}=, 021\right)$.

Las personas que expresan alegría $(12,9 \%)$ presentan elementos dinámicos en su entorno (insectos, sol, nubes blancas), pero no pasa lo mismo con el resto de emociones, siendo esta diferencia significativa $\left(X^{2}=, 015\right)$. En esta misma línea, se observan que los menores que expresan alegría $(1,6 \%)$ y tristeza $(3,3 \%)$ no dibujan un entorno hostil, en cambio los menores que expresan miedo $(1,6 \%)$ y rabia $(1,6 \%)$ no dibujan un entorno afable, apreciándose diferencias significativas $\left(X^{2}=, 038\right)$ entre las diferentes emociones.

- Diferencias respecto la emoción auténtica

Otro dato de especial interés y altamente significativo $\left(\mathrm{X}^{2}=, 000\right)$ indica que los menores que expresan miedo $(22,6 \%)$ como emoción parásita, en realidad sienten rabia como emoción auténtica. Los menores que expresan tristeza (14,5\%) como emoción parásita sienten miedo auténtico y los menores que expresan rabia $(29 \%)$ como emoción parásita sienten miedo como emoción auténtica.

También se encuentran diferencias significativas en cuanto a los mecanismos de defensa $\left(X^{2}=, 040\right)$, señalando que las personas con rabia auténtica (28\%) y miedo auténtico $(31,7 \%)$ dibujan espinas en su rosal. Pero no lo hacen las personas con tristeza $(10 \%)$ por lo que no se protegen.

\section{Discusión}

La primera coincidencia en los dos estudios es que el GC formado por población general presenta alegría como emoción parásita. Esto puede deberse a la necesidad de la persona de satisfacer las expectativas del entorno, buscando continuamente el bienestar subjetivo, la satisfacción vital y la felicidad. Autores como Cantón y Justicia (2008) indican que haber sufrido abuso sexual infantil supone una alta probabilidad de padecer problemas de depresión. Curiosamente las personas que presentan abuso y las personas con depresión han dibujado el rosal con colores poco fieles a la realidad. Este hallazgo puede ser de especial interés en investigaciones futuras para la detección de la depresión en menores.

En cuanto la variable sexo, los hombres muestran rabia como emoción parásita, dibujan más espinas en el rosal y ninguna hoja. En cambio, las mujeres presentan tristeza, no dibujan espinas, pero si hojas. Las hojas son elementos de intercambio con el entorno y suelen indicar las capacidades comunicativas de la persona. Las espinas son las defensas de la persona que pueden ser elementos de protección o de defensa. Parece interesante observar como estos datos coinciden con los aprendizajes emocionales recibidos a través de nuestra cultura.

En ambos estudios, la alegría se presenta de forma afable, la tristeza en forma de vacío, y el miedo y la rabia se presentan de forma hostil. Aunque en los menores el miedo aparece más de forma agresiva y en los adultos en forma de protección. La manera como dibujan el entorno también puede ser un dato relevante para identificar la emoción parásita en torno a la que se construye el problema.

Respecto a la emoción auténtica ambos estudios coinciden en que las personas 
con miedo parásito sienten rabia como emoción auténtica, los que expresan tristeza presentan miedo auténtico y los que expresan rabia sienten miedo. Por tanto, reforzamos nuestra hipótesis de la importancia de identificar las emociones auténticas para poder satisfacer las necesidades emocionales y contribuir a un mayor bienestar (Lizeretti, 2012).

Además, actuar desde la emoción auténtica facilita estrategias de afrontamiento. En este sentido, podemos observar como en relación a la presencia de espinas, tanto la rabia como el miedo auténtico dibujan espinas en su rosal pero el miedo lo hace en forma de defensa y la rabia en forma de protección. Las personas con tristeza no ponen espinas en su rosal, por lo que tienen más dificultades para afrontar de forma eficaz sus conflictos.

En el estudio 1, un dato interesante al comparar grupos diagnósticos indica que las personas con depresión presentan tristeza como emoción parásita y en la ansiedad miedo. Estos resultados coinciden con los criterios diagnósticos establecidos según los manuales utilizados (DSM y CIE). Pero resulta interesante cuando se analiza la emoción auténtica, que se prohíbe, que varía en relación a la emoción expresada, siendo el miedo el que más prevalece en la depresión y en el TAG, y la rabia en la agorafobia. Este dato puede ser de especial relevancia no solo para la explicación y la comprensión del proceso emocional en diferentes psicopatologías sino para una adecuada intervención basada en la satisfacción de las necesidades vitales de la persona.

Asimismo, las personas con depresión se presentan de forma más desconectada, dibujan en la parte superior de la hoja y utilizan colores poco realistas. Estos indicadores coinciden con los modelos cognitivos más relevantes de la literatura científica. Por ejemplo en el modelo de Beck (1967) se indica que existen diferentes elementos clave para el desarrollo de la depresión. En primer lugar, la tríada cognitiva, formada por una visión negativa sobre uno mismo, el mundo y el futuro; en segundo lugar, los pensamientos automáticos negativos que desencadenan la activación emocional; en tercer lugar, las distorsiones sistemáticas en el procesamiento de la información que nos llega desde nuestro medio; y en cuarto lugar, la disfunción en los esquemas cognitivos, que son formas de percibir la realidad que incluyen creencias y emociones. Por otro lado, otro factor altamente relacionado con la depresión es la rumiación, es decir un tipo de pensamiento negativo repetitivo que incapacita a la persona a la búsqueda de soluciones (Nolen-Hoeksema y Morrow, 1991). Esto se podría explicar por la gran dificultad que tienen las personas con depresión para identificar con claridad de sus estados emocionales y de repararlos de forma adecuada (para una revisión véase Lizeretti y Rodriguez, 2011).

Las personas con depresión tampoco dibujan espinas, por lo que se sienten desprotegidos, pero dibujan elementos de protección en el entorno. Abramson, Seligman y Teasdale (1978) indican que las personas más propensas a sufrir depresión son aquellas que cuando experimentan situaciones que no pueden controlar activan respuestas emocionales negativas y atribuyen su indefensión a 
causas internas, globales y estables. En esta misma línea algunos estudios demuestran que el estilo atribucional de las personas indefensas se relaciona con los síntomas depresivos, siendo el proceso emocional un aspecto fundamental en la depresión (Camuñas, 2005).

En cambio, los pacientes con ansiedad se muestran más conectados con la realidad, tienden a dibujar en el centro de la hoja o en la parte de abajo y utilizan colores más realistas. Esto podría relacionarse con los pensamientos o síntomas obsesivos que se asocian a estos trastornos.

Otro dato de interés es como las personas con TAG dibujan espinas que les permiten protegerse y tienen como emoción auténtica el miedo. En cambio, las personas con agorafobia dibujan las espinas en forma defensiva y presentan rabia prohibida, dato que confirma la importancia de intervenir en relación a las necesidades vitales auténticas (Lizeretti, 2009; 2012).

En relación al estudio 2, los menores tutelados tienen rabia parásita y disponen de menos recursos que el CG, lo que viene indicado por un entorno desnutrido u hostil. Estos indicadores confirman el malestar general que presentan y la dificultad que tienen para gestionar sus emociones como consecuencia de todos los cambios que han sufrido ya a edades tan tempranas. En la mayoría de casos provienen de entornos negligentes y de maltrato, proceden de familias desestructuradas donde han quedado desatendidas sus necesidades básicas tanto físicas como afectivas. Este entorno hostil puede provocar en el menor una inestabilidad emocional que frecuentemente se manifiesta a través de conductas agresivas, de descontrol o de dificultad para mantener relaciones sanas. De hecho, diferentes autores señalan la alta prevalencia de trastornos emocionales y conductuales en este ámbito (e.g., Del Valle, Saneiro y Bravo, 2011).

El segundo dato relevante a destacar es la alta prevalencia de maltrato y abuso sexual, lo que genera una serie de consecuencias en los menores que repercuten directamente en su ajuste psicosocial, en sus relaciones de apego, en su autoestima, en sus expectativas de control y en su capacidad empática (Drapeau, Saint Jacques, Lépine, Begin y 2007; Haskett, Nears, Sabourin y McPherson, 2006; Lázaro y López, 2010).

Los menores que han sufrido abuso sexual pueden sentir rechazo hacia el entorno y conductas de retraimiento o por otro lado presentar mucha ansiedad, que expresan con sentimientos de tristeza o rabia. Aquellos que han sufrido maltrato físico y/o psicológico suelen presentar actitudes agresivas, destructivas, rebeldes e hiperactividad relacionadas con la emoción de la rabia.

En relación a la emoción parásita también se observan algunos indicadores interesantes como que las personas con alegría parásita no dibujan en la parte izquierda de la hoja, por lo que parecen menos focalizados en el pasado. En cambio las personas con tristeza tienen dificultad para focalizar su atención en el aquí y ahora, por lo que no dibujan el rosal en el centro. Las personas con miedo focalizan mucho la atención hacia el futuro, aspecto relevante dado la coincidencia entre la 
expresión del miedo y la anticipación de amenazas futuras manifestada con conductas evitativas, fóbicas, etc.

Otro dato que sustenta la interpretación de la técnica es como las personas que presentan alegría dibujan elementos dinámicos, como sol, insectos, nubes... que complementan su dibujo. Estos elementos están relacionados con nuestras relaciones de intercambio con el exterior y con cómo nos sentimos respecto al mundo que nos rodea.

Finalmente, se puede concluir que la mayoría de los resultados confirman la hipótesis de que contrariamente a lo que se cree los pacientes con ansiedad tienen como emoción prohibida la rabia y los pacientes con depresión el miedo. Esta técnica podría servir para detectar la presencia de alguna forma de abuso en los menores. Asimismo, hay coincidencia entre los dos estudios en cuanto a la relación entre emociones parásitas y auténticas y su forma de protegerse del entorno, confirmándose algunos de los sesgos culturales más frecuentes en nuestra cultura emocional. Además, los datos obtenidos también contribuyen a explicar algunos aspectos que se "captan intuitivamente", y que un mayor conocimiento emocional a través de la Inteligencia Emocional puede ayudar a descifrar.

Futuras investigaciones deben ir orientadas a la mejora de la sistematización de los datos, definiendo y acotando mejor las categorías y ampliando la muestra para consolidar los resultados encontrados. Dadas las relaciones encontradas en los dos estudios, también sería interesante correlacionar la técnica con síntomas y otros resultados psicométricos. Así como extender el análisis a otros ámbitos de intervención o comparar resultados de intervención pre y post tratamiento. Por todo ello es importante seguir avanzando en la investigación empírica en este ámbito a fin de poder demostrar y consolidar la eficacia de las técnicas proyectivas y de fantasía guiada.

\section{Referencias Bibliográficas}

Abramson, L.Y., Seligman, M.E.P y Teasdale, J.D. (1978). Learned helplessness in humans. Critique and reformulation. Journal of Abnormal Psychology, 87, 49-74.

Augusto-Landa, J. M., López-Zafra, E., Martínez de Antoñana, R., y Pulido Martos, M. (2006). Perceived emotional intelligence and life satisfaction among university teachers. Psicothema, 18, 152-157.

Beck, A.T. (1967). Depression: Clinical, experimental and theoretical aspects. Nueva York: Harper y Row.

Brackett, M. A. y Mayer, J. D. (2003). Convergent, discriminant and incremental validity of competing measures of emotional intelligence. Personality and Social Psychology Bulletin, 29(9), 1-12.

Bracket, M. A., Mayer, J. D. y Warner, R. M. (2004). Emotional intelligence and its relation to everyday behavior. Personality and Individual Differences, 36, 1387-1402.

Camuñas, N. (2005). Dimensiones atribucionales asociadas a la depresión. EduPsykhé, 4(2), 179-197.

Cantón, D. y Justicia, F.(2008). Afrontamiento del abuso sexual infantil y ajuste psicológico a largo plazo. Psicothema, 20, 509-515.

Ciarrochi, J., Chan, A., y Caputi, P. (2000). A critical evaluation of the emotional intelligence construct. Personality and Individual Differences, 28, 539-561.

Davis, S.K., y Humphrey, N. (2012). The influence of emotional intelligence (EI) on coping and mental health in adolescence: Divergent roles for trait and ability EI. Journal of Adolescence, 35(5), 1369-1379. 
Del Valle, J., Saneiro, A., y Bravo, A. (2011). Salud Mental de menores en acogimiento residencial. Guía para la prevención e intervención en hogares y centros de protección de la Comunidad Autónoma de Extremadura. Extremadura: Junta de Extremadura.

Downey, L. A., Mountstephen, J., Lloyd, J., Hansen, K., y Stough, C. (2008). Emotional intelligence and scholastic achievement in Australian adolescents. Australian Journal of Psychology, 60(1), 10-17.

Drapeau, S., Saint Jacques, M.C., Lépine, R., Begin, G., y Bernard M. (2007) Processes that contribute to resilience among youth in foster care. Journal of adolescence, 30, 977-999.

Extremera, N., y Fernández-Berrocal, P. (2004). Inteligencia Emocional, calidad de las relaciones interpersonales y empatía en estudiantes universitarios. Clínica y Salud, 15, 117-137.

Fernández-Berrocal, P., Extremera, N y Ramos, N. (2004): Validity and reliability of the V Spanish modified version of the Trait Meta-Mood Scale. Psychological Reports, 94, 751-755.

Gohm, C.L. y Clore, G.L. (2002). Tour latent traits of emotional experience and their involvement in well-being, coping, and attributional style. Cognition and Emotion, 16, 495-518.

Haskett, M. E., Nears, K., Sabourin, C., y McPherson, A. (2006). Diversity in adjustment of maltreated children: Factors associated with resilient functioning. Clinical Psychology Review, 26, 796-812.

Hertel, J., Schütz, A., y Lammers, C. (2009). Emotional intelligence and mental disorder. Journal of Clinical Psychology, 65(9), 942-954..

Jacobs, M., Snow, J., Geraci, M., Vythilingam, M., Blair, R. J., Charney, D. S., Pine, D. S., y Blair, K. S. (2008). Association between level emotional intelligence and severity of anxiety in generalized social phobia. Journal of Anxiety Disorders, 22(8), 1487-1495.

Keyes, C.L.M., y López, S.J. (2002). Toward a science of mental health: Positive directions in diagnosis and interventions. Londres: Oxford University Press.

Kokkinos, C. M., y Kipritsi, E. (2012). The relationship between bullying, victimization, trait emotional intelligence, self-efficacy and empathy among preadolescents. Social Psychological Education, 15, 42-58.

Lázaro, S. y López, F (2010) Continuidad de los efectos del maltrato durante la infancia en adolescentes acogidos en los centros de protección. Infancia y Aprendizaje, 33(2) 255-268.

Lizeretti, N. P. (2009). Tratamiento de los trastornos de ansiedad: Diseño y evaluación de una intervención grupal basada en la Inteligencia Emocional. Barcelona: Universitat Ramon Llull (Tesis doctoral). Recuperado de http://www.tesisenred.net/bitstream/handle/10803/9269/Tesis_Nathalie_P_Lizeretti.pdf?sequence=1.

Lizeretti, N. P. (2012). Terapia basada en inteligencia emocional. Manual de tratamiento. Lleida: Milenio.

Lizeretti, N. P., y Extremera, N.(2011) Emotional intelligence and clinical symptoms in outpatients with generalized anxiety disorder (GAD). Psychiatric quarterly, 82(3), 253-260.

Lizeretti, N. P., y Rodríguez, A. (2011). La inteligencia emocional en salud mental: Una revisión. Ansiedad y Estrés, 17(2-3), 233-253.

Lizeretti N., Extremera N., y Rodríguez A. (2012). Perceived emotional intelligence and clinical symptoms in mental disorders. Psychiatric Quarterly, 83(4),407-18.

Lizeretti, N.P. y Gimeno-Bayón, A. (2014). Inteligencia emocional y valores éticos. Revista de Psicoterapia 25(97), 79-104. http://revistadepsicoterapia.com/inteligencia-emocional-y-valores-eticos.html

Lomas, J., Stough,C., Hansen, K., y Downey, L.A. (2012). Brief report: Emotional intelligence, victimisation and bullying in adolescents. Journal of Adolescence, 35. 207-211.

Mayer, J. D. y Salovey, P. (1997). What is Emotional Intelligence? Nueva York: Basic Books.

Mayer, J. D., Caruso, D. y Salovey, P. (1999). Emotional intelligence meets traditional standards for and intelligence. Intelligence, 27, 267-298.

Mayer, J. D., Roberts, R. D., y Barsade, S. G. (2008). Human abilities: Emotional intelligence. Annual Review of Psychology, 59, 507-536.

Moriarty, N., Stough, C., Tidmarsh, P., Eger, D., y Dennison, S. (2001). Deficits in emotional intelligence underlying adolescent sex offending. Journal of Adolescence, 24, 1-9.

Nolen-Hoeksema, S., y Morrow, J. (1991). A prospective study of depression and posttraumatic stress symptoms after a natural disaster: The 1989 Loma Prieta earthquake. Journal of Personality and Social Psychology, $61,115-121$.

Perna, G., Menotti, R., Borriello, G., Cavedini, P., Bellodi, L., y Caldirola, D. (2010). Emotional intelligence in panic disorder. Rivista di Psichiatria, 45, 320-325.

Rosal, R. (1983). Investigación sobre la creatividad y psicología humanista. Revista de Psiquiatría y Psicología Humanista, 4, 1-15.

Rosal, R. (2002). El poder psicoterapéutico de la actividad imaginaria y su fundamentación científica. Barcelona, España: Instituto Erich Fromm de Psicoterapia Integradora Humanista. 
Salguero, J. M., y Iruarrizaga, I. (2006). Relaciones entre inteligencia emocional percibida y emocionalidad negativa: ansiedad, ira y tristeza/depresión. Ansiedad y estrés, 12(2-3), 207-221.

Salovey, P., Stroud, L.R., Woolery, A., y Epel, E.S. (2002). Perceived emotional intelligence, stress reactivity, and symptom reports: Further explorations using the Trait Meta-Mood Scale. Psychology and Health, 17, 611627.

Schutte, N., Malouff, J., Hall, L., Haggerty, D., Cooper, J., Golden, C., y Dornheim, L. (1998). Development and validation of measure of emotional intelligence. Personality and Individual Differences, 25, 167-177.

Stevens, J.O. (1976). El darse cuenta. Sentir, vivenciar e imaginar. Santiago de Chile, Chile: Cuatro Vientos. 